\title{
Conocimiento del profesor de secundaria de la práctica matemática en clases de geometría
}

\section{Knowledge of practices in mathematics of a secondary teacher in geometry classes}

\section{Diana Zakaryan, ${ }^{1}$ Leticia Sosa ${ }^{2}$}

\begin{abstract}
Resumen: Los estudios sobre el significado de la especialización en el conocimiento del profesor de matemáticas están tomando un papel cada vez más importante en el campo de la investigación en educación matemática. Este artículo tiene por objetivo destacar tal especialización del conocimiento del profesor de matemáticas, desde el modelo Mathematics Teachers' Specialized Knowledge (MTSK) y desde las prácticas inherentes a la propia matemática, aportando a la comprensión y caracterización del conocimiento de la práctica matemática a partir de los datos empíricos. A través de observación de aula y entrevista a una profesora de matemáticas de enseñanza secundaria, hemos identificado el conocimiento del profesor del papel de los símbolos y de las convenciones matemáticas; del papel de las demostraciones y de sus principales métodos, del significado y del rol de la condición necesaria y suficiente, y destacamos el conocimiento de estrategias heurísticas de resolución de problemas y del papel de la generalización. Concluimos poniendo de relieve la
\end{abstract}

Fecha de recepción: 23 de mayo de 2020; Fecha de aceptación: 18 de octubre de 2020.

1 Instituto de Matemáticas, Pontificia Universidad Católica de Valparaíso, Chile, diana.zakaryan@pucv. cl, orcid.org/0000-0002-3400-8399

2 Unidad Académica de Matemáticas, Universidad Autónoma de Zacatecas, México, Isosa@uaz.edu.mx, orcid.org/0000-0002-4905-6684 
importancia de estos conocimientos del profesor para favorecer el desarrollo de las capacidades de hacer matemáticas de los alumnos.

Palabras clave: Conocimiento especializado del profesor de matemáticas, práctica matemática, geometría, profesor de matemáticas, enseñanza secundaria.

\begin{abstract}
Studies about the implication of specialization in a mathematics teacher's knowledge are taking important role in the field of mathematics education research. From this perspective, the goal of this research is to study the knowledge of the practices in mathematics of the Mathematics Teachers' Specialized Knowledge model (MTSK), contributing to understanding and characterization of the nature of this teacher knowledge. Through empirical data from classroom observation and an interview with a high-school mathematics teacher we have been identified the teacher's knowledge of the role of symbols and mathematical conventions; knowledge about the importance of proofs and their principal methods, of the meaning and the role of necessary and sufficient conditions; equally, knowledge of heuristic strategies of problem solving and the role of generalization. We conclude by highlighting the importance of this teacher's knowledge to favor the development of students' abilities to do mathematics.
\end{abstract}

Keywords: Mathematics teachers' specialized knowledge, practices in mathematics, geometry, mathematics teacher, high-school level.

\title{
INTRODUCCIÓN
}

El conocimiento profesional de los profesores de matemáticas incluye el "contenido que abordan durante la lección, pero es evidente que los profesores tienen que tener conocimientos adicionales para poder enseñar las matemáticas de manera apropiada a los alumnos" (Bromme, 1994, p. 73). Los estudios sobre el conocimiento del profesor están tomando un papel cada vez más central en la investigación en educación matemática y, la búsqueda de lo que significa la especialización en el conocimiento del profesor de matemáticas, se ha ido convirtiendo en una empresa cada vez más importante en el campo de la investigación (Scheiner, Montes, Godino, Carrillo, y Pino-Fan, 2017). Estudios recientes han abordado este tema al describir e identificar facetas o tipos de conocimiento del 
profesor que han sido considerados cruciales para la enseñanza de las matemáticas, y han tratado de obtener evidencias empíricas para su respaldo (e.g. Ball, Thames, y Phelps, 2008; Carrillo, Climent, Contreras, y Muñoz-Catalán, 2013; Rowland, Huckstep, y Thwaites, 2005).

Por otra parte, es reconocido que la actitud de los profesores hacia el conocimiento matemático es necesariamente diferente de la de los matemáticos investigadores (Davis y Renert, 2013). Según Ball (2000), las investigaciones matemáticas están orientadas hacia el empaquetamiento de sus descubrimientos en formulaciones estrictas (teorías, teoremas, fórmulas, etc.), mientras la labor del profesor se orienta hacia el desempaquetamiento de los mismos. Todo esto exige conocimientos matemáticos específicos del profesor. Pero, ¿̇uál es la naturaleza de estos conocimientos?

En la literatura especializada acerca del conocimiento profesional del profesor de matemáticas, se da importancia al conocimiento de la comprensión de la naturaleza de la disciplina, el conocimiento sobre las matemáticas (Ball, 2003), que se refiere a una gran cantidad de conocimientos sobre las matemáticas, por ejemplo, los principales desacuerdos dentro de la disciplina (tanto en el pasado como en el presente), cómo se justifican y validan las afirmaciones, qué implica hacer y participar en el discurso del área (Ball y McDiarmid, 1990). Este es el conocimiento sintáctico (Schwab, 1978) de las matemáticas que considera el significado de los debates filosóficos dentro de la disciplina y el conocimiento de las formas en que se introduce, valida y acepta el nuevo conocimiento dentro de una comunidad académica (e.g., Grossman, Wilson, y Shulman, 2005). Con base en estas ideas, en el modelo Mathematics Teacher's Specialized Knowledge (MTSK), se define el conocimiento de la práctica matemática (KPM) como el conocimiento de cualquier actividad matemática Ilevada a cabo sistemáticamente que representa un pilar de la creación matemática y que conforma una base lógica de la cual se pueden extraer reglas (Carrillo et al., 2018).

A pesar de la importancia de ese conocimiento, son escasos los estudios que han profundizado en la naturaleza del KPM (e.g. Delgado y Zakaryan, 2018) y, por otra parte, las investigaciones desarrolladas con el MTSK con profesores de distintos niveles educativos reportan escasas evidencias de aspectos del KPM en sus clases de matemática (e.g. Zakaryan y Sosa, 2019).

Concordamos con Godino (2009) en que es útil contar con modelos que permitan un análisis más detallado de cada uno de los tipos de conocimiento que los docentes ponen en juego en la enseñanza de las matemáticas, contribuyendo así a los aspectos analíticos y teóricos de la investigación en Educación 
Matemática. En este sentido, el objetivo de este estudio es describir el conocimiento de la práctica matemática que se manifiesta en el aula a partir de evidencias empíricas, contribuyendo a la comprensión y caracterización de la naturaleza de este conocimiento del profesor.

\section{FUNDAMENTOS TEÓRICOS}

\section{CONOCIMIENTO ESPECIALIZADO DEL PROFESOR DE MATEMÁtICAS}

Una de las contribuciones más significativas de los estudios sobre el conocimiento de los profesores de matemáticas ha sido la propuesta del modelo MKT (Ball et al, 2008). El modelo especifica el conocimiento de los docentes, representa un importante punto de partida para presentar a la comunidad e implicar especialización en el conocimiento de los docentes, específicamente en uno de los tres subdominios del conocimiento matemático, el conocimiento especializado del contenido (SCK). Sin embargo, hay propuestas, como la de Carrillo et al. (2018), en la cual se supone que la especialización del conocimiento de los profesores de matemáticas es específica tanto para el conocimiento matemático como para el conocimiento didáctico del contenido de las matemáticas. Entendemos el conocimiento especializado en términos de Scheiner et al. (2017), donde la especialización en el conocimiento del profesor de matemáticas significa estilo de conocimiento en lugar de un tipo de conocimiento, es decir, el conocimiento es visto como un todo orgánico y holístico que emerge en una estructura dinámica situada y adaptada al contexto en el cual el profesor realiza su práctica docente.

El modelo Mathematics Teachers' Specialized Knowledge - MTSK (Carrillo et al., 2013) adopta una postura crítica ante la tendencia de comparar el conocimiento del profesor de matemáticas con el conocimiento exigido a otros profesionales, como matemáticos o profesores de asignaturas distintas de las matemáticas (Scheiner et al., 2017) y considera el carácter especializado del conocimiento del profesor de matemáticas que abarca seis subdominios del conocimiento (figura 1). Tres de ellos conforman el Conocimiento Didáctico del Contenido: el conocimiento de la enseñanza de las matemáticas, el conocimiento de las características del aprendizaje de las matemáticas y el conocimiento de los estándares de aprendizaje de las matemáticas. Otros tres se consideran en el Conocimiento Matemático: el conocimiento de los temas, el 
conocimiento de la estructura de las matemáticas y el conocimiento de la práctica matemática. EI MTSK incluye también el dominio de las creencias/ concepciones del profesor sobre la matemática y su enseñanza y aprendizaje. Su ubicación en el centro del modelo expresa la estrecha relación con los subdominios anteriores.

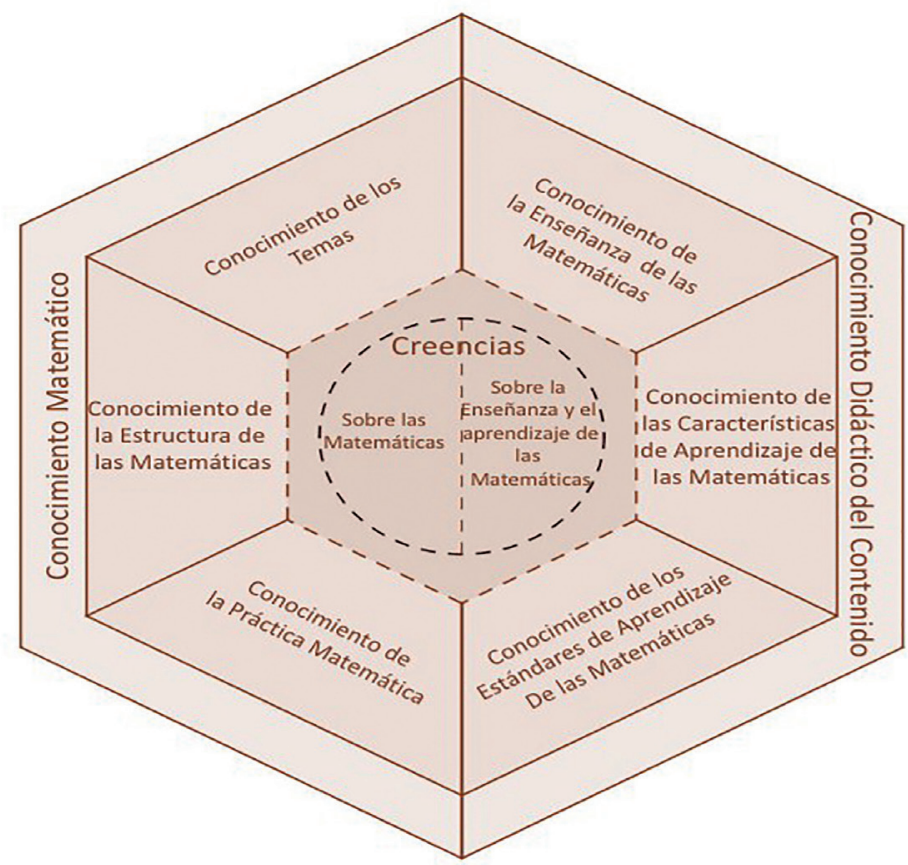

Figura 1. Subdominios del Modelo MTSK (Sosa, Flores-Medrano y Carrillo, 2016, p. 154).

Dado el interés de este estudio en uno de los subdominios del Conocimiento Matemático, describimos brevemente los tres subdominios que lo componen. De acuerdo con Carrillo et al. (2018), el Conocimiento de los Temas (KoT) se refiere a qué y de qué manera conoce el profesor de matemáticas los tópicos que enseña, es decir, al conocimiento del profesor de los procedimientos involucrados en un tópico, de los registros de representación, de la fenomenología (incluye usos y aplicaciones) asociada al tópico abordado, de las definiciones (incluye imágenes y ejemplos de objetos matemáticos) y de las 
propiedades y sus principios, y en sí, al conocimiento profundo del contenido matemático y sus significados. El Conocimiento de la Estructura de las Matemáticas (KSM) está constituido por el conocimiento del profesor en cuanto a las conexiones interconceptuales entre tópicos matemáticos, distinguiendo cuatro tipos de conexiones: de complejización (se relaciona con el contenido matemático posterior, la matemática elemental desde un punto de vista avanzado), de simplificación (la matemática avanzada se contextualiza retrospectivamente en el contenido matemático más elemental sobre el que se basa), auxiliares (participación necesaria de un elemento matemático en procesos más amplios) y transversales (cuando diferentes elementos del contenido matemático tienen características en común). Finalmente, el Conocimiento de la Práctica Matemática (KPM) incluye el conocimiento acerca de cómo se crea, se hace y se produce matemáticas (en este último subdominio se profundizará en la siguiente sección).

Como se puede apreciar, el Conocimiento Matemático contiene conocimientos que guardan relación directa con la organización interna de la matemática: temas (que incluye los entes matemáticos), conexiones entre entes matemáticos y formas de hacer o construir matemáticas. En este sentido, dentro del KPM se destaca la importancia de que el profesor no sólo conozca los resultados matemáticos establecidos sino las formas de proceder y pensar en matemáticas para llegar a ellos (e.g. Delgado-Rebolledo y Zakaryan, 2020). El KPM aporta la idiosincrasia de la matemática como rama del saber, como actividad humana.

\section{CONOCIMIENTO DE LA PRÁCTICA MATEMÁTICA}

Los autores del modelo MTSK adoptan una definición operativa de conocimiento, entendida como un conjunto de recursos disponibles para que el profesor los use con el fin de resolver problemas, alcanzar metas y desarrollar tareas; este conocimiento no es necesariamente correcto (Schoenfeld, 2010). Esta última consideración permite al investigador profundizar en la comprensión del conocimiento y del desempeño de los docentes. Además, el término "práctica" juega un papel importante en la investigación en el área de educación matemática. La práctica generalmente se refiere a las acciones o actividades que ocurren en el proceso de enseñanza-aprendizaje (e.g., Carrillo et al., 2018), particularmente, en el ámbito de la capacitación docente y el desarrollo profesional podemos encontrar la mejor práctica (Lampert, 2010), práctica central (Grossman, 
Hammerness y McDonald, 2009), prácticas de alto aprovechamiento (Ball, Sleep, Boerst y Bass, 2009), en cuanto a aprender a enseñar.

En este artículo, práctica matemática significa el funcionamiento de las matemáticas en lugar del proceso de enseñanza:

[...] como cualquier actividad matemática llevada a cabo sistemáticamente, que representa un pilar de la creación matemática y que se ajusta a una base lógica de la cual se pueden extraer reglas. Entre muchas otras cosas, el conocimiento del profesor de matemáticas sobre esta práctica incluye saber cómo demostrar, justificar, definir, hacer deducciones e inducciones, dar ejemplos y comprender el papel de los contraejemplos. También incluye una comprensión de la lógica que sustenta cada una de estas prácticas; en resumen, se refiere a lo que se puede llamar un conocimiento sintáctico (Schwab, 1978) de las matemáticas. (Carrillo et al., 2018, pp. 9-10)

Según estos autores, es necesario que el profesor de matemáticas tenga este conocimiento; así, tal conocimiento proporciona estructuras de pensamiento lógico que ayuda a comprender el funcionamiento de diversos aspectos de la matemática. A su vez, esto ayuda a robustecer el propio conocimiento del profesor y le permite negociar el razonamiento matemático que los estudiantes ponen en juego para aceptar, refutar o refinarlo.

Algunos ejemplos del conocimiento de la práctica matemática son el conocimiento acerca de cómo validar el conocimiento en matemáticas; que la prueba es una herramienta de demostración reconocida en matemáticas (e.g., Balacheff, 2000) la que puede tener diferentes significados en diferentes contextos institucionales y en las diversas formas argumentativas que se ponen en juego en las clases de matemáticas (e.g., Godino y Recio, 2001); el conocimiento del papel y la importancia de la prueba en matemáticas (e.g., Oehrtman y Lawson, 2008); de los roles de los ejemplos y contraejemplos en el proceso de validación (e.g., Götte, Renzulli y Scaglia, 2010; Lakatos, 1976); y la funcionalidad de las convenciones matemáticas y de sus posibles explicaciones en la generación y validación del conocimiento matemático (e.g., Martínez, 2005; Poincaré, 1984). Asimismo, el conocimiento del profesor de las heurísticas y las estrategias de resolución de problemas (e.g., Polya, 1945; Schoenfeld, 1992) o determinar las características de las definiciones de conceptos matemáticos, las estructuras subyacentes de las definiciones y el proceso de creación de una definición (e.g., Van Dormolen y Zaslavsky, 2003; Zaslavsky y Shir, 2005; Zazkis y Leikin, 2008). Por último, saber el papel de la abstracción y la generalización 
en la creación y el reconocimiento de patrones y modelos, también es parte del subdominio KPM.

De acuerdo con Davis y Renert (2013), consideramos que el conocimiento que necesitan los profesores no es simplemente un conjunto de conceptos básicos claramente definidos y bien conectados, sino una mezcla sofisticada de diversas realizaciones de conceptos matemáticos y conciencia de los complejos procesos a través de los cuales se producen las matemáticas. En este sentido asumimos el conocimiento del profesor como complejo, integral y relacionado y, con fines analíticos tratamos de caracterizarlo, proporcionando evidencias de cómo vive el conocimiento de la práctica matemática de un profesor de matemáticas de nivel secundaria en el aula.

\section{MÉTODO}

A partir de un estudio de caso (Stake, 1995) desde el enfoque interpretativo, indagamos acerca del conocimiento de una profesora de matemáticas (en adelante Eva) del décimo grado (alumnos entre 14-15 años) de una escuela secundaria pública. La selección del caso se ha regido por las características académicas de Eva. Eva ha sido la egresada con el mejor promedio de su promoción de la carrera de Pedagogía en Matemática y posee un Magíster en Didáctica de la Matemática. En el momento del estudio, Eva contaba con tres años de experiencia en aula y formaba parte de un grupo de Lesson Study (Isoda y Olfos, 2009) en su escuela, en el que los profesores se reúnen periódicamente para reflexionar sobre los contenidos matemáticos, situaciones matemáticamente críticas y sus prácticas docentes con el fin de mejorarlas en la acción. Este hecho hace alusión al carácter dinámico, evolutivo y complejo del conocimiento de Eva, visto como una disposición participativa para aprender (Charalambous y Pitta-Pantazi, 2016).

\section{RECOLECCIÓN Y ANÁLISIS DE DATOS}

Los datos recolectados para este estudio provienen de las grabaciones de las clases de Eva, a través de la técnica observación no participante (Cohen y Manion, 2007). Elegimos un tema de geometría dado que esperamos que proporcione situaciones más ricas a las de otros dominios de la matemática 
escolar en términos de la práctica matemática (por ejemplo, demostración). Las diez sesiones (90 minutos cada una) dedicadas al contenido de semejanza de triángulos (e.g. elementos básicos asociados con el concepto de triángulo, aplicación del teorema de Thales para determinar medidas desconocidas, criterios de similitud y su aplicación) fueron audio y videograbadas y posteriormente transcritas y divididas, según el objetivo matemático de la profesora, en episodios - unidades de análisis que corresponden a las comunicaciones orales y escritas de la profesora y de sus alumnos. Los datos obtenidos se han tratado a partir de análisis de contenido (Bardin, 1997), por dos investigadores quienes codificaron las unidades de análisis de acuerdo con las nociones de práctica matemática encontradas en la revisión de la literatura (por ejemplo, validación o papel de los símbolos).

Con el fin de ampliar el enfoque basado en la práctica (e.g., Charalambous y Pitta-Pantazi, 2016), enriqueciendo las oportunidades para acceder al conocimiento del profesor, los datos se complementaron con una entrevista semiestructurada con Eva. Del mismo modo, varias investigaciones sobre el KPM de los profesores de matemáticas Ilaman la atención sobre su escasa aparición en el aula y sugieren abordarlo buscando evidencia de ello en otros escenarios y/o mediante otros instrumentos de recopilación de datos (e.g., Montes, 2014). Dicha diversidad permite no solo el refinamiento de los primeros pasos de análisis, sino también facilita la triangulación de diferentes fuentes de datos (Stake, 1995), logrando una consistencia interna y externa. La entrevista de 70 minutos de duración se basó en una guía de preguntas relacionadas con los descriptores de KPM que se han encontrado en el análisis de los videos para confirmar y complementarlo. El análisis de la transcripción de la entrevista (a través del análisis de contenido), de este modo, se centró en la búsqueda de evidencias de su manifestación.

\section{RESULTADOS Y DISCUSIÓN}

En esta sección se presentan los descriptores del KPM emergentes de los datos empíricos, procedentes de las observaciones y la entrevista. La presentación de los resultados obedece a la organización de las evidencias de acuerdo a la aparición de los descriptores, más que al orden de sesiones de clases, por ello, cuando las evidencias observadas en distintas sesiones se referían al mismo descriptor, estas se agruparon para fortalecer y enriquecer la discusión. 
En la primera sesión, donde el objetivo es recordar los conceptos elementales de geometría asociados a triángulos, Eva solicita a los alumnos trabajar en una guía, identificando elementos y características de los triángulos. En el siguiente extracto (una parte del episodio), Eva muestra a los alumnos cómo se puede escribir simbólicamente que dos triángulos son congruentes:

1284 Eva: Congruencia también tiene un símbolo que se ocupa, y eso no lo habíamos mencionado,

1285 Io vamos a agregar. Ese es un signo igual ¿cierto? [escribe =] Para la congruencia

1286 se utiliza eso, pero se utiliza con este simbolito arriba [escribe @]. Con esa curvita.

1287 Es un símbolo que se utiliza para decir que dos triángulos, dos figuras, son congruentes.

1288 Siempre tratamos de utilizar símbolos matemáticos para ahorrarnos las palabras.

Tal como se puede observar, Eva manifiesta el conocimiento del uso de los símbolos " $=$ " y " $\cong$ " para igual y congruente respectivamente [1284-1286], y menciona que son para "ahorrarnos las palabras" [1288]. El hecho de que Eva conozca los símbolos " $=$ " $\mathrm{y}$ " $\cong$ " indica su conocimiento incluido en el KoT (se trata de parte de unas notaciones asociadas a la congruencia entre figuras), mientras que, al destacar el papel de los símbolos para expresar la información abreviada y precisa, da cuenta de conocimiento que se incluye en KPM.

En la entrevista, a la pregunta: ¿Cuál es el papel de los símbolos en matemáticas?, responde de la siguiente manera, confirmando su idea anteriormente expuesta:

Eva: Bueno, los símbolos matemáticos se usan para reducir el lenguaje y para que puedan relacionar también conceptos matemáticos, diferenciarlos de lenguaje natural, en este caso... Identificar los conceptos matemáticos, para no confundirlos y diferenciarlos. Por ejemplo, en el caso de semejanza, usar el símbolo de los triángulos, no es para otro elemento, usar el símbolo de semejanza no significa otra cosa ...

Para reducir el lenguaje, identificar conceptos, entender el lenguaje matemático.

Eva indica que los símbolos matemáticos se usan para reducir el lenguaje y, teniendo en cuenta el episodio anterior, se aprecia su conocimiento de las funciones principales de los símbolos en matemáticas, mencionadas por Davis y Hersh (1989), a saber, designar con precisión y claridad, y abreviar, señalando que sin el proceso de abreviación el discurso matemático difícilmente sería 
posible. Por otra parte, se observa su conocimiento en cuanto a que el lenguaje matemático está formado por símbolos y se les asigna un significado preciso y peculiar (e.g. Alcalá, 2002), así como el conocimiento de que los símbolos permiten la interpretación y comprensión de las ideas matemáticas de forma tal que cada símbolo sea claro y sin ambigüedades (Davis y Hersh, 1989). De este modo, podemos identificar el siguiente descriptor del conocimiento de Eva:

KPM1: Conocimiento del papel de los símbolos para reducir y expresar abreviadamente la información, identificar conceptos y entender el lenguaje matemático.

En el siguiente extracto de la primera sesión, Eva cuestiona a los alumnos respecto a cómo pueden saber si el ángulo presentado en la figura es un ángulo recto:

194 Eva: ¿Por qué es un ángulo recto? ¿Por qué podríamos saberlo en esa figura?

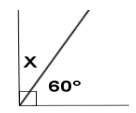

195 Por el cuadradito dice Al. Por el símbolo que ahí aparece, usted puede asumir que

196 eso es un ángulo recto. Si ese símbolo no está, usted no lo podría asumir. Porque

197 podría ser que valiera $91^{\circ}$, y la imagen visual es casi la misma.

Con la pregunta que plantea y la respuesta que da, se evidencia su conocimiento de que uno puede asumir ciertas características de objetos matemáticos si están explícitamente enunciadas a través de símbolos matemáticos convencionales. En este caso, muestra conocimiento de que "el cuadradito" asegura que, en el dibujo, el ángulo sea recto y que no se puede fiar solamente de la imagen visual, dando cuenta de su conocimiento de papel de los símbolos en contextos de validación. Asimismo, en la segunda sesión, mientras identifican los ángulos formados entre dos rectas paralelas y la tercera que las intersecta, Eva manifiesta el conocimiento de la misma índole, anteriormente mencionada, cuando llama la atención de los alumnos de que solo pueden asumir que dos rectas son paralelas cuando en las condiciones se dice explícitamente o se da el símbolo // "dos slash". 
373 Eva: Si tuviéramos dos rectas que en la figura no se juntan. Es más, si estas líneas a la 374 vista parecieran paralelas, si yo no lo indico, no lo puede asumir, aunque lo parezcan.

375 Si yo le doy esa información, porque ese es el símbolo de paralelas, dos slashs. Si 376 yo digo que esas dos son paralelas, son paralelas. Si no lo digo, no lo puede asumir.

Análogamente, se observa el conocimiento de Eva del papel de los símbolos para asegurar que un enunciado es verdadero en el fragmento de la entrevista que sigue:

Eva: Justamente se busca que ellos validen su conocimiento. Como no tienen instrumentos de medición, una representación que no tenga ese símbolo no representa un ángulo recto necesariamente. Igual que les digo con las rectas paralelas. Ustedes pueden observar que estas líneas son paralelas, pero no podemos comprobar, por tanto, si no me lo dice el enunciado, yo no puedo asumir que son paralelas.

En este fragmento, corrobora el conocimiento evidenciado en la clase, pues menciona que, si ciertas características no están explícitamente enunciadas a partir de símbolos matemáticos convencionales, no se podría asumirlas ("una representación que no tenga ese símbolo no representa un ángulo recto necesariamente"). Es decir, en matemática una constatación sobre un dibujo no es suficiente para probar que un enunciado es verdadero (e.g. Götte, Renzulli, y Scaglia, 2010).

Por otra parte, a la pregunta acerca del "papel de la convención matemática", da la siguiente respuesta:

Eva: A pesar de que la convención a veces es un poco molesta, uno no sabe bien cómo fundamentarla, son como cimientos para algunos conocimientos. Para los alumnos no es tan fácil, porque uno les lleva por el camino de que todo es demostrable, que son capaces de encontrar razonamiento para decir que algo es cierto. Mientras la convención viene como para decir: "Tú, esto acéptalo". Y uno puede decir que los matemáticos tuvieron que decidir así, porque convenía, de ahí viene la palabra.

En este fragmento de la entrevista, Eva muestra su conocimiento del papel de las convenciones en matemáticas, que concuerda con Poincaré (1984) cuando sostiene que estas son principios científicos que no son ni evidencias, ni generalizaciones experimentales, ni hipótesis planteadas a manera de conjetura con 
la intención de ser verificados. La elección entre todas las convenciones posibles es regida por la experimentación, pero es libre, y solo atiende la necesidad de evitar cualquier contradicción. Se aprecia su conocimiento de que una convención matemática es una conveniencia para las matemáticas, que, en este caso, ha tomado forma de una definición (e.g. Martínez, 2005), cuando menciona que "son fundamentales para construir un conocimiento posterior", dando lugar al siguiente descriptor:

KPM2: Conocimiento del papel de las convenciones en matemáticas y particularmente, el papel de símbolos convencionales en contextos de validación.

En la segunda sesión, donde el objetivo es identificar los ángulos formados entre dos rectas paralelas y la tercera que las intersecta, Eva explica:

377 Eva: ...Sólo se cumple cuando hay rectas paralelas, y cuando no coinciden. Ahí dice,

378 no coincidentes. Si no hay rectas paralelas, usted no puede decir que esa relación

379 se cumple.

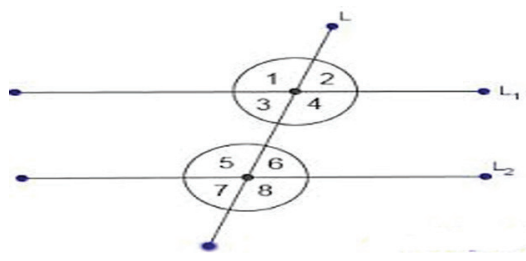

Por ejemplo, si tuviéramos dos rectas, que en la figura no se juntan, ¿̇cierto? De

380 hecho, a simple vista, uno nota que se podrían cruzar. Si yo dibujo eso así [dibuja

381 rectas no paralelas], ¿la relación de arriba se cumple? ¿Sí o no? ¿Cuándo se cumple

382 esta relación?

383 Al: Cuando son paralelas.

384 Eva: Cuando las líneas son paralelas.

En este extracto, Eva muestra el conocimiento de que para poder afirmar algún supuesto en matemáticas o deducir otras propiedades de un objeto matemático, deben cumplirse las condiciones necesarias y/o suficientes, en este caso, apelando a la condición necesaria ("solo se cumple cuando hay rectas paralelas") para que se dieran las equivalencias entre los ángulos formados. Este es el conocimiento del significado de la proposición "si A entonces B" (e.g. Solow, 
1993), donde A es la hipótesis de que las rectas son paralelas y B es la conclusión de que los ángulos formados son equivalentes.

En la entrevista, se le preguntó a Eva: ¿Qué significa condición necesaria y condición suficiente?:

Eva: Una condición necesaria es algo que debe pasar sí o sí para que un teorema se cumpla. Y una condición suficiente es algo que basta que se cumpla para que uno pueda decir que el teorema se cumple. Por ejemplo, un problema puede tener distintas características, pero tal vez no son todas necesarias para afirmar que algo sucede ahí. Entonces, ¿qué se cumple ahí?, basta que se cumpla este, pero una condición necesaria es algo que tiene que pasar sí o sí.

Asimismo, se puede apreciar su conocimiento del significado de la condición suficiente ("basta que cumpla") y que "A implica B, no necesariamente significa que B implica A".

Por otra parte, en la séptima sesión, donde el objetivo es aplicar el teorema de Thales para determinar medidas de segmentos y ángulos, Eva hace una observación, llamando la atención de los alumnos e insistiendo en que se dieran cuenta de cómo funciona una condición suficiente y necesaria.

2122 Eva: Si hay rectas paralelas, los ángulos correspondientes son de igual medida.

2123 ¿Y funciona al revés? ¿Qué significa que esa expresión funcione al revés? Si

2125 tenemos paralelas, hay ángulos de igual medida. Si hay rectas paralelas, los ángulos

2126 correspondientes son iguales cंcierto? Pero ahora, si por ejemplo, yo hiciera esa

2129 recta, no sé si esa recta es paralela, pero lo que sí sé que esos ángulos son iguales,

2130 ipodré concluir que esa recta es paralela? [Si]. A eso nos referimos.

2133 Puedo ocuparlo partiendo de las paralelas o puedo ocuparlo partiendo de los ángulos 2134 ¿Sí? Entonces aquí vamos a poner "viceversa".

En este caso, se observa el conocimiento de Eva del rol de la condición suficiente y necesaria en un teorema: se parte de una condición (suficiente) para concluir con una sentencia y cuando se da esa sentencia ocurre la misma condición (necesaria). En otras palabras, se aprecia su conocimiento de que la proposición "A es equivalente a B" o que "A si y solo si B" significa demostrar que A implica 
B y B implica A (e.g. Solow, 1993). De este modo, se identifica el siguiente descriptor del conocimiento de Eva:

KPM3: Conocimiento del significado y rol de las condiciones necesaria y suficiente en un teorema.

En la tercera sesión, Eva ha tenido por objetivo que los alumnos clasifiquen triángulos dados (algunos vienen dados por medidas de sus lados, otros de sus ángulos, otros de ambas medidas o sin ninguna de las dos), según las características comunes que identifiquen.

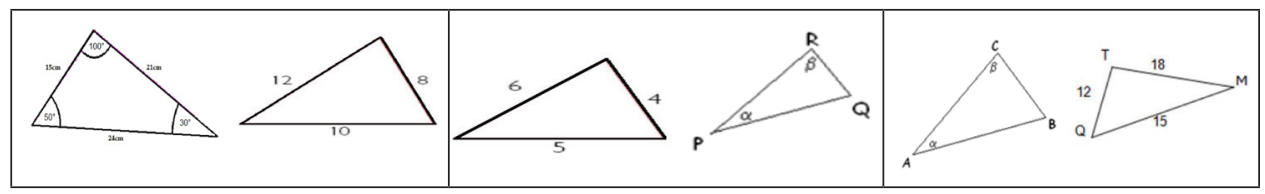

Los alumnos llegan a la conclusión de que todos los triángulos dados son escalenos, a pesar de que haya dos triángulos que no se indican las medidas de sus lados ni de sus ángulos. En el siguiente extracto, Eva trata de que los alumnos se den cuenta de ese hecho:

635 Eva: ¿̇Todos son escalenos? Hay un punto ahí. Si yo tengo el dato de cuánto miden los 636 lados, probablemente podré saber si son o no escalenos, pero si no tienen la medida, 637 ipuedo afirmar que son escalenos? [No].

638 Podrían ser isósceles, y si no tenemos las medidas no lo podemos asegurar.

Por otra parte, en la entrevista, a la pregunta: ¿Cómo se valida en matemáticas y cuándo un razonamiento es aceptado como válido?, responde lo siguiente:

Eva: Con demostraciones, claramente. Demostrando, con la misma teoría matemática, puedo decir que algo es válido para el contexto que estoy trabajando... Entonces validarlo, es efectivamente recurrir a esa teoría matemática construida y hay cosas para avanzar, pero hay cosas construidas y verificando que tenga lógica, que tenga coherencia con lo que estamos planteando, que sea válido con lo que se define... Entonces, ese razonamiento se va validando a medida que voy encontrando elementos matemáticos que lo sustenten. Podría yo equivocarme, podría tener una hipótesis, pero necesito validarla con la teoría matemática. 
En este extracto se puede observar el conocimiento de Eva de que una forma de validar en matemáticas es a través de prueba ("demostrando, con la teoría matemática misma, puedo decir que algo es válido"), es decir, la prueba es una herramienta para validar en matemáticas (e.g., Balacheff, 2000). Además, muestra conocimiento de que, en matemáticas, el razonamiento se acepta como válido en la medida en que esté respaldado por elementos matemáticos aceptados ("ese razonamiento se va validando a medida que voy encontrando elementos matemáticos que lo sustenten"). También, muestra el conocimiento de que, para debatir, en matemáticas, se apoya en propiedades y definiciones claramente establecidas y aceptadas (e.g, Götte et al., 2010), dando lugar al siguiente descriptor:

KPM4: Conocimiento de que la afirmación de algún supuesto, en matemáticas, debe sustentarse en propiedades enunciadas y aceptadas.

En la séptima sesión, cuando los alumnos establecen algunas razones entre los segmentos formados, Eva muestra cómo se comprueba la proporcionalidad entre dos razones $\frac{4,5}{x}=\frac{4}{3}$, recuerda el método de producto cruzado $4,5 \times 3=4 \times$ y justifica detalladamente el procedimiento de "pasar" el 3 y x al "otro lado" a partir de la multiplicación por inversos de ambas partes de la expresión. En el extracto que sigue, Eva destaca que no es necesario cada vez hacer estos procedimientos porque hay un teorema que los fundamenta.

2037 Eva: ¿Para qué? Para que se entienda que yo puedo pasar, directamente, de este puedo pasar a 2038 ese [se refiere de una expresión a otra]. No necesitan hacer eso entremedio, porque hay un 2039 teorema de proporciones que lo fundamenta ¿sí? Y ese teorema se fundamenta con lo que 2040 nosotros hicimos. Utilizar los inversos.

En este episodio, Eva evidencia el conocimiento de que una vez demostrado un teorema, se garantiza su generalización a otros casos con condiciones similares, resaltando de esa manera la aplicación de la generalización a casos similares [2038-2040].

En la entrevista corroboramos este conocimiento, preguntándole: ¿Para qué sirve generalizar en matemáticas y cuándo se puede generalizar?. Respondió: 
Eva: Bueno, la generalización sirve para establecer un concepto, para definirlo, cंverdad? Y poder validar un conocimiento en cierto contexto. Entonces llevar un caso particular a un caso general permite no tener que demostrar caso a caso y entender que esto lo que estoy demostrando tiene cierta lógica que se cumple para todos los elementos que cumplan ciertas condiciones. Entonces, generalizar permite ahorrar ese trabajo de exploración y decir que cada vez que me enfrento a esto va a pasar esto.

En este fragmento, se observa el conocimiento de Eva de que la generalización sirve para generar definiciones matemáticas ("para establecer un concepto, para definirlo") y permite pasar de un caso particular a otro más general ("llevar un caso particular a un caso general permite no tener que demostrar caso a caso..."). De este modo, se evidencia su conocimiento de que una vez demostrado un teorema sus conclusiones se generalizan para otros casos bajo las condiciones similares, como una regla general (e.g. Castro, Cañadas, y Molina, 2010), es decir, que los teoremas son generalizaciones para algún conjunto de objetos matemáticos que cumplen ciertas características. De este modo, se identifica el siguiente descriptor:

KPM5: Conocimiento del papel de la generalización en matemáticas.

La novena sesión se sigue con la aplicación del teorema de Thales. Eva propone a los alumnos resolver un problema y les deja unos minutos para el trabajo individual.

2977 Eva: [Dicta] Problema: En un mapa la distancia entre dos ciudades es de $20 \mathrm{~cm}$. Se sabe que 2978 la distancia real entre ellas es de 200kms.1.¿Cuántas veces más grande es la distancia 2979 real, respecto a la del mapa? 2. ¿Cuál es la razón entre ambas distancias? 3: ¿Cuál será la 2980 distancia real entre otras dos ciudades que se encuentran a $13 \mathrm{~cm}$ en el mismo mapa?

Posteriormente, escribe en la pizarra las respuestas que obtuvieron algunos alumnos para discutirlas. En el extracto siguiente, Eva, escribe una de las respuestas erróneas de los alumnos, en la cual dividen dos cantidades con diferentes unidades de medida (centímetros entre kilómetros). Su objetivo es que los alumnos se den cuenta de por qué la respuesta es incorrecta. 
3038 Eva: Entonces, habían respondido la primera pregunta... y su respuesta había sido 10, ¿cierto? 3039 ¿Es correcta esa respuesta? [Si].

3040 ¿Qué operación había hecho la compañera para obtener ese 10? [Dividir 200 en 20].

3041 Pero, ¿efectivamente es 10 veces más grande la distancia? O sea, que si tengo $1 \mathrm{~cm}$ en el

3042 mapa, según eso, ¿̇cuánto debería medir en la realidad? [10].

3043 ¿10 qué? Centímetros, es 10 veces más grande. Si algo mide 20cm, según eso,

3044 si el $20 \mathrm{~cm}$ lo hacemos 10 veces más grande ¿̇uánto conseguimos? ¿Conseguimos

$3045200 \mathrm{~km}$ ? [No].

3046 ¿Cuánto conseguimos? Si esto fuera cierto, $1 \mathrm{~cm}$ va a equivaler a 10 en la realidad, ¿o no?

3047 Porque es 10 veces más grande. Y ¿̇cuánto es 20 por 10? [200].

3048 ¿200 qué? En este caso, centímetros. ¿Obtengo kilómetros al hacer esto? [No].

3049 Es un error de comparación, porque si usted se devuelve, no va a obtener jamás $200 \mathrm{~km}$.

En este extracto, se puede observar cómo, mediante una prueba, suponiendo que la respuesta fuese correcta [3046-3048], Eva, muestra a los alumnos el absurdo de tal respuesta [3048-3049] y la imposibilidad de obtener la condición dada $(200 \mathrm{~km})$. Aunque no se trata de una demostración formal, se aprecia el razonamiento de un método de demostración (prueba por contradicción): para demostrar la invalidez de una proposición, se supone como punto de partida que la proposición es cierta; si la derivación final es una contradicción, se concluye que la proposición original es falsa y el argumento es inválido (e.g. Solow, 1993). De este modo, se observa el conocimiento de Eva del papel de la prueba en matemáticas (e.g. Oehrtman y Lawson, 2008).

Por otra parte, en la entrevista, Eva responde a la pregunta: ¿Qué métodos de demostración conoce?, de la siguiente manera:

Eva: Por contradicción, directa, ya no me acuerdo los nombres. Está por la negación: negando la afirmación y comprobando que no puede ser. La directa... no, no recuerdo más.

Si bien, su respuesta es escueta y, como ella misma reconoce, no recuerda los nombres de métodos de demostración, muestra conocer el método directo, por contradicción y por contraposición ("negación"). De acuerdo con el extracto de la clase y de la entrevista, se identifica el siguiente descriptor: 
KPM6: Conocimiento del método de prueba por contradicción para validar/ demostrar en matemáticas.

En la novena sesión, donde el objetivo es resolver problemas aplicando el teorema de Thales, Eva plantea un problema [2575-2578] y da algunas indicaciones para proceder en su resolución:

2575 Eva: Problema: Durante el día, se observa que un poste de luz proyecta una sombra de 6 metros

2576 sobre el suelo, y que la sombra de una persona parada cerca del poste es de 3 metros (las

2577 sombras proyectadas en la misma dirección). Si la persona mide 1,7 metros, ¿̇cuánto

2578 medirá el poste? Primer paso, va a intentar hacer un dibujo de la situación, eso es lo

2579 primero. Va a volver a leer el problema y va a imaginárselo y hacer un dibujo de eso.

2580 Supongamos que tenemos acá el poste, asumimos que está perpendicular al suelo.

2581 Y se dice que hay una persona que está cerca del poste. El piso está acá, la persona podría

2582 estar acá [hace el dibujo]. ¿¿ué datos daba el problema? ¿Cómo podemos relacionarlos?

En este extracto, se observa su conocimiento de heurísticas para resolver problemas. Eva, sugiere que, en un primer paso, los alumnos traten de imaginar y dibujar la situación [2578-2580]; es decir, se manifiesta su conocimiento de estrategias de resolución, particularmente que el esquema o dibujo son soportes relevantes para entender el problema (e.g. Polya, 1945). Asimismo, plantea preguntas que ayudan a entender el problema y orientar la solución: ¿qué datos daba el problema?, ¿cómo podemos relacionarlos?

Cuando, en la entrevista, se le pregunta a Eva: ¿Qué hace cuando está frente a un problema desafiante?, responde:

Eva: Bueno, tomar camino de representaciones, tratar de dibujar algo. Podría tomar datos del problema y hacer cálculos. Podría directamente identificar elementos matemáticos que están dando vuelta ahí, representarlos de una manera algebraica. Podría tratar de abstraerme del contexto del problema y ver el contexto matemático y resolverlo y volverme al contexto del problema, para ver si tiene sentido o no. Puedo recurrir al ensayo y error, tantear y luego tratar de formalizar eso que estoy probando. Y si no entiendo todo el contexto, voy a tomar lo que comprendo y voy a tratar de hacer algo con eso. Podría ir de lo simple a lo complejo o de lo abstracto a lo concreto también, o al revés. 
Como se puede observar en el fragmento de la entrevista, Eva, muestra su conocimiento de estratégicas heurísticas de resolución de problemas, que en términos de Polya (1945), constituirían las fases del proceso de resolución de problemas. Por ejemplo, se aprecia la fase entender el problema ("tomar camino de representaciones, tratar de dibujar, imaginarse lo que sucede en el problema..."), la fase hacer un plan ("extraer la información y ver a qué se relaciona, recurrir al ensayo y error, ir de lo simple a lo complejo o de lo abstracto a lo concreto"), la fase mirar hacia atrás ("volverme al contexto del problema para ver si tiene sentido o no"). De ahí, se identifica el siguiente descriptor:

KPM7: Conocimiento de algunas estrategias heurísticas de resolución de problemas matemáticos.

De este modo, los resultados de la investigación dan cuenta del conocimiento de Eva de distintas prácticas matemáticas. Así, el KPM1 y KPM2 surgen cuando la profesora destaca el papel de los símbolos y convenciones matemáticas y sugieren una comunicación en y/o sobre las matemáticas, la cual, de acuerdo con Davis y Hersh (1989), requiere de un lenguaje común (lenguaje matemático, en este caso) que permite la interpretación y comprensión de las ideas matemáticas que se comparten. Por tanto, para que la actividad matemática sea posible, es necesario conocer el significado de estos símbolos para saber cómo interpretarlos y usarlos adecuadamente. En este sentido, Zakaryan y Sosa (2019) concluyen que el uso del lenguaje matemático es una de las prácticas que es relevante desde la educación de la primera infancia, donde la parte importante es utilizar el lenguaje matemático de una manera, aunque no fuese completa, pero necesariamente correcta. Por otro lado, en los niveles más avanzados, se observa la necesidad de comprender el significado de los símbolos matemáticos, así como su uso correcto.

Por otra parte, los descriptores KPM3, KPM4 y KPM6 se refieren a distintas formas de validar el saber en matemáticas. Estos descriptores sugieren distinguir entre una condición necesaria y una condición suficiente y conocer sus significados, saber cuál es la verdad en matemáticas y saber que lo que constituye una "demostración" puede tener diferentes significados en los diferentes contextos institucionales y diversas formas argumentativas que se ponen en juego en las aulas de matemáticas (e.g., Godino y Recio, 2001). Por tanto, aunque es necesario justificar o dar demostraciones en la educación preescolar, primaria y secundaria, es importante enfatizar que, en la comunidad escolar, es difícil trabajar desde los cánones de aceptabilidad compartidos por la comunidad de matemáticos. Por lo tanto, es necesario "aliviar" las demandas si se espera tener 
algún éxito en la producción de justificaciones para las conjeturas enunciadas (Mariotti, 2006). En este caso, hemos visto que Eva ha encontrado algunas formas de "aliviar" estas demandas (por ejemplo, pruebas no formales) sin perder de vista su significado matemático.

Finalmente, los descriptores KPM5 y KPM7 caracterizan el quehacer matemático, revelando aspectos que se usan para trabajar genéricamente en matemáticas, a partir de las generalizaciones y usando diferentes estrategias heurísticas en la resolución de problemas. La comunidad de educación matemática reconoce la importancia del conocimiento del profesor y el uso de estrategias heurísticas (e.g., Polya, 1945; Schonfield, 1992). Por otro lado, varios autores (e.g., Mason, Graham y Johnston-Wilder, 2005) destacan la importancia del conocimiento del profesor sobre la generalización como una actividad empírica inductiva para difundir la conciencia de la actividad de generalizar y promoverla como la esencia de la matemática, para que ocurra el pensamiento matemático en los estudiantes.

Cabe señalar que, en este documento, solo se presentaron los descriptores del KPM que se evidenciaron en las clases de Eva y se corroboraron en la entrevista, ya que el interés del estudio está en caracterizar el KPM de Eva que se manifiesta en acción. Este hecho significa que se han obtenido, además, descriptores de KPM que solo se identificaron en la entrevista, por ejemplo, algunos en relación al conocimiento de Eva del papel de los ejemplos y contraejemplos en el proceso de validación matemática, al conocimiento de las características de una definición matemática y al conocimiento del papel de la abstracción en las matemáticas, que son otros aspectos del KPM.

\section{CONCLUSIONES}

Este estudio ha pretendido caracterizar cómo este tipo de conocimiento matemático se manifiesta en el aula y de este modo contribuir al campo de la investigación en educación matemática, aportando a su comprensión. La especialización, en el enfoque adoptado en el MTSK, atañe al modelo en su conjunto y la profundización en la caracterización del KPM aporta especificidad en relación con la matemática.

Los descriptores emergentes del estudio representan un aporte teórico, permitiendo acentuar conocimientos acerca de las características inherentes a la práctica matemática, necesarios para consolidar su propio conocimiento, para 
saber negociar los razonamientos matemáticos dados por sus alumnos. Asimismo, estos descriptores ponen de relieve la importancia del conocimiento del profesor para favorecer el desarrollo de las capacidades de hacer matemáticas de los alumnos (Abrantes, 2001), reconocidas a nivel internacional a partir de diferentes aspectos que dan cuenta de ello (e.g. promover el razonamiento matemático, la resolución de problemas, la importancia de justificar, conjeturar, probar, generalizar, etc.), dichos aspectos son requeridos de una u otra forma a los estudiantes de acuerdo a evaluaciones y estándares internacionales (e.g. OECD, 2014; NCTM, 2000).

El conocimiento del profesor acerca de la elección de los símbolos (convencionales o no convencionales) y su uso coherente en diferentes contextos, así como el conocimiento del papel del lenguaje matemático, le permite comunicar las ideas matemáticas de manera abreviada y con precisión respecto al significado estricto de los términos.

Destacamos la importancia del conocimiento del profesor de las distintas formas de validar en matemáticas: que el profesor conozca el papel que juegan las demostraciones, los métodos de demostración, distinguir una condición necesaria y una suficiente. Además, saber cómo funciona la demostración en distintos niveles educativos, cómo se valida en ellos el conocimiento matemático, qué negociaciones deben establecerse con los alumnos al respecto, qué criterios de demostración son aceptados en distintos niveles y cómo van evolucionando de un nivel a otro.

Por último, el conocimiento de diferentes heurísticas de resolución de problemas y del papel de la generalización permiten al profesor hacer más tangible y consciente las forma en que se desarrolla la solución del problema y extender un determinado método a otras situaciones (e.g. Polya, 1945).

Los datos obtenidos empíricamente permiten apreciar que una importante cantidad de descriptores del KPM de Eva no se ha manifestado en el aula, sino solo en la entrevista, confirmando la complejidad de evidenciar el KPM solo a partir de las observaciones de aula (e.g. Montes, 2014) y dando origen a varias preguntas acerca de la naturaleza de ese conocimiento y de cómo se refleja en la práctica docente. Por ejemplo, si bien a través de la entrevista la profesora mostró conocer otros aspectos característicos del KPM no apreciados en el aula, cabe preguntarse por qué no se dieron las situaciones en las cuales ese conocimiento pudiese ponerse en juego.

A pesar de que existe un reconocimiento del papel del profesor para fomentar la cultura de las matemáticas en el aula (e.g. Stylianides, 2007; Yackel y Cobb, 
1996), de la comprensión del profesor de matemáticas para que ocurra un verdadero pensamiento matemático en el aula $(\mathrm{Ma}, 1999)$ y de la importancia de que un profesor de matemáticas conozca distintos tipos de razonamientos y sepa en qué contextos matemáticos unos son más adecuados que otros (e.g. Ball y McDiarmid, 1990; Ball y Bass, 2009; Rowland et al, 2009), poco se menciona sobre cómo formar a los profesores para que estos aspectos tengan vida en el aula. Es preciso que los estudios en educación matemática avancen en los planteamientos acerca de dónde y cuándo los profesores de matemáticas adquieren el conocimiento de la práctica matemática o cómo los propios profesores experimentan el hacer matemáticas.

\section{AGRADECIMIENTOS}

Esta investigación se ha realizado bajo el financiamiento del proyecto FONDECYT N 11140092 de la Comisión Nacional de Investigación Científica y Tecnológica de Chile; proyecto VRIEA-PUCV 039.439/2020. Las autoras del artículo son miembros de la "Red Iberoamericana MTSK", reconocida por la Asociación Universitaria Iberoamericana de Postgrado (AUIP).

\section{REFERENCIAS}

Abrantes, P. (2001). Mathematical competence for all: Options, implications and obstacles. Educational Studies in Mathematics, 47, 125-143.

Alcalá, M. (2002). La construcción del lenguaje matemático. Graó.

Balacheff, N. (2000). Procesos de prueba en los alumnos de matemáticas. Una Empresa Docente. Universidad de los Andes.

Ball, D. L., y McDiarmid, G. W. (1990). The subject-matter preparation of teachers. In W.

R. Houston and M. H. J. Sikula (Eds.), Handbook of research on teacher education (pp. 437-449). Macmillan.

Ball, D. L. (2000). Bridging practices: Intertwining content and pedagogy in teaching and learning to teach. Journal of Teacher Education, 51(3), 241-247.

Ball, D. L. (2003). What mathematical knowledge is needed for teaching mathematics? Secretary's Summit on Mathematics.

Ball, D., Thames, M. H., y Phelps, G. (2008). Content knowledge for teaching: What makes it special? Journal of Teacher Education, 59(5), 389-407. 
Ball, D., y Bass, H. (2009). With an eye on the mathematical horizon: Knowing mathematics for teaching to learners' mathematical futures. In The 2009 Curtis Center Mathematics and Teaching Conference. University of California.

Ball, D., Sleep, L., Boerst, T. A. y Bass, H. (2009). Combining the development of practice and the practice of Development in Teacher Education. The Elementary School Journal, 109(5), 458-474.

Bardin, L. (1997). Content analysis. Livraria Martins Fontes.

Bromme, R. (1994). Beyond subject matter: A psychological topology of teachers' professional knowledge. In R. Biehler, R. W. Scholz, R. Strässer y B. Winkelmann (Eds.), Mathematics didactics as a scientific discipline: The state of the art (pp. 73-88). Kluwer.

Carrillo, J., Climent, N., Contreras, L. C., y Muñoz-Catalán, M. C. (2013). Determining Specialised Knowledge for Mathematics Teaching. In B. Ubuz, C. Haser, y M. A. Mariotti (Eds.), Proceedings of the CERME 8 (pp. 2985-2994). Middle East Technical University.

Carrillo, J., Climent, N., Contreras, L. C., Montes, M., Escudero-Ávila, D., y Flores-Medrano, E. (2014). (Eds.), Un Marco Teórico para el Conocimiento Especializado del Profesor de Matemáticas [A Theoretical Framework for the Mathematics Teacher's Specialized Knowledge]. Universidad de Huelva Publicaciones.

Carrillo, J., Climent, N., Montes, M., Contreras, L.C., Flores-Medrano, E., Escudero-Ávila, D., ... y Muñoz-Catalán, M. (2018). The mathematics teacher's specialised knowledge (MTSK) model, Research in Mathematics Education, 20(3), 1-18. http://doi.org/10.108 0/14794802.2018.1479981

Castro, E., Cañadas, M. C., y Molina, M. (2010). El razonamiento inductivo como generador de conocimiento matemático. Uno, 54, 55-67.

Cohen, L. y Manion, L. (2002). Métodos de investigación educativa. La Muralla.

Charalambous, C., y Pitta-Pantazi, D. (2016). Perspectives on priority mathematics education: Unpacking and understanding a complex relationship linking teacher knowledge, teaching, and learning. In L. English y D. Kirshner (Eds.), Handbook of international research in mathematics education (pp. 19-59). Routledge.

Davis, Ph., y Hersh, R. (1989). Experiencia Matemática. [The Mathematical Experience]. Editorial Labor.

Davis, B., y Renert, M. (2013). Profound understanding of emergent mathematics: broadening the construct of teacher' disciplinary knowledge. Educational Studies in Mathematics, 82(2), 245-265.

Delgado-Rebolledo, R., y Zakaryan, D. (2018). Knowledge of the practice in mathematics in university teachers. En V. Durand-Guerrier, R. Hochmuth, S. Goodchild, y N.M. Hogstad (Eds.), Proceedings of the Second Conference of the International Network 
for Didactic Research in University Mathematics (pp. 393-402). University of Agder and INDRUM.

Delgado-Rebolledo, R., y Zakaryan, D. (2020). Relationships between the Knowledge of Practices in Mathematics and the Pedagogical Content Knowledge of a Mathematics Lecturer. International Journal of Science and Mathematics Education, 18(3), 567-587. https://doi.org/10.1007/s10763-019-09977-0

Godino, J. D. (2009). Categorías de análisis de los conocimientos del profesor de matemáticas. Unión: Revista Iberoamericana de Educación Matemática, 20, 13-31.

Godino, J. D., y Recio, A. M. (2001). Significados institucionales de la demostración. Implicaciones para la educación matemática. Revista Enseñanza de las Ciencias, 19(3), 405-414.

Götte, M., Renzulli, F., y Scaglia, S. (2010). El contraejemplo en la producción de conjeturas de propiedades geométricas. Revista de Educación Matemática. https://revistas. unc.edu.ar/index.php/REM/article/view/10256

Grossman, P., Wilson, S., y Shulman, L. (2005). Profesores de sustancia: el conocimiento de la materia para la enseñanza. Profesorado. Revista de currículum y formación del profesorado, 9(2), 1-24.

Grossman, P., Hammerness, K., y McDonald, M. (2009). Redefining teaching, re-imagining teacher education. Teachers and Teaching, Theory and Practice, 15(2), 273-289.

Isoda, M., y Olfos, R. (2009). El enfoque de resolución de problemas en la enseñanza de la matemática a partir del estudio de clases. Ediciones Universitarias de Valparaíso, Pontifica Universidad Católica de Valparaíso.

Lakatos, I. (1976). Proofs and refutations: The logic of mathematical discovery. Cambridge University Press.

Lampert, M. (2010). Learning teaching in, from, and for practice: What do we mean? Journal of Teacher Education, 61(1-2), 21-34.

Ma, L. (1999). Knowing and teaching elementary mathematics: teachers' understanding of fundamental mathematics in china and the United States. Erlbaum.

Mariotti, M. A. (2006). Proof and proving in mathematics education. En A. Gutierréz y P. Bolero (Eds.), Handbook of research on the psychology of mathematics education: Past, present and future (pp. 173-204). Sense Publishers.

Martínez, G. (2005). Los procesos de convención matemática como generadores de conocimiento. Revista Latinoamericana de Investigación en Matemática Educativa, 8(2), 195-218.

Mason, J., Graham, A., y Johnston-Wilder, S. (2005). Developing thinking in algebra. The Open University y Paul Chapman Publishing. 
Montes, M. (2014). Conocimiento especializado del profesor de matemáticas acerca del infinito. Un estudio de caso. (Tesis de doctorado no publicada). Universidad de Huelva, España.

National Council of Teachers of Mathematics [NCTM] (Ed.). (2000). Principles and standards for school mathematics (Vol. 1). National Council of Teachers of Mathematics.

Oehrtman, M., y Lawson, A. E. (2008). Connecting science and mathematics: The nature of proof and disproof in science and mathematics. International Journal of Science and Mathematics Education, 6(2), 377-403.

Organization for Economic Co-operation and Development [OECD]. (2014). PISA 2012 results in focus: what 15-year-olds know and what they can do with that they know. Author.

Poincaré, H. (1984). Filosofía de la ciencia. Serie Nuestros Clásicos No. 32. Universidad Nacional Autónoma de México.

Polya, G. (1945). How to solve it. University Press.

Rowland, T., Huckstep, P., y Thwaites, A. (2005). Elementary teachers' mathematics subject knowledge: the knowledge quartet and the case of Naomi. Journal of Mathematics Teacher Education, 8, 255-281.

Rowland, T., Turner, F., Thwaites, A., y Huckstep, P. (2009). Developing primary mathematics teaching. Reflecting on practice with the knowledge quartet. SAGE Publications Ltd.

Stylianides, A. J. (2007). Proof and proving in school mathematics. Journal for Research in Mathematics Education, 38(3), 289-321.

Scheiner, T., Montes, M. A., Godino, J. D., Carrillo, J., y Pino-Fan, L. (2017). What Makes Mathematics Teacher Knowledge Specialized? Offering Alternative Views. International Journal of Science and Mathematics Education. http://doi.org/10.1007/s10763017-9859-6

Schoenfeld, A. H. (1992). Learning to think mathematically: Problem Solving, Metacognition and Sense-Making in Mathematics. En D. Grouws (Ed.), Handbook for Research on mathematics Teaching and Learning (pp. 103-107). Macmillan.

Schoenfeld, A. H. (2010). How we think. Routledge.

Schwab, J. (1978). Education and the structure of the disciplines. In I. Westbury y N. J. Wilkof (Eds.), Science, curriculum, and liberal education (pp. 229-272). University of Chicago Press.

Solow, D. (1993). Cómo entender y hacer demostraciones en matemáticas. Editorial Limusa.

Sosa, L., Flores-Medrano, E. y Carrillo, J. (2016). Conocimiento de la enseñanza de las matemáticas del profesor cuando ejemplifica y ayuda en clase de álgebra lineal. Educación Matemática, 28(2), 151-174.

Stake, R. E. (1995). The art of case study research. Sage. 
Van Dormolen, J., y Zaslavsky, O. (2003). The many facets of a definition: The case of periodicity. Journal of Mathematical Behavior, 22(1), 91-106. http://doi.org/10.1016/ S0732-3123(03)00006-3

Yackel, E., y Cobb, P. (1996). Sociomathematical norms, argumentation, and autonomy in mathematics. Journal for Research in Mathematics Education, 27(4), 458-477.

Zakaryan, D., y Sosa, L. (2019). ¿Cómo los profesores hacen prácticas matemáticas en sus aulas? En R. Olfos, E. Ramos, y D. Zakaryan (Eds.), Formación docente: Aportes a la práctica docente desde la Didáctica de la Matemática (pp. 281-300). Graó.

Zaslavsky, O., y Shir, K. (2005). Students' Conceptions of a Mathematical definition. Journal for Research in Mathematics Education, 36(4), 317-346.

Zazkis, R. y Leikin, R. (2008). Exemplifying definitions: a case of a square. Educational Studies in Mathematics, 69(2), 131-148. http://doi.org/10.1007/s10649-008-9131-7

\section{DIANA ZAKARYAN}

Dirección: Pontificia Universidad Católica de Valparaíso

Blanco Viel 596, 2380000

Valparaíso, Chile

Teléfono: $\quad 569322274009$ 